\title{
The Center of Mathematical Sciences and Applications at Harvard University
}

\author{
by Shing-Tung Yau, Director ${ }^{*}, \dagger$
}

The Center of Mathematical Sciences and Applications at Harvard University was created as a fusion point for mathematics, statistics, physics, and related sciences, with the purpose of establishing applied mathematics at Harvard as a first-class, interdisciplinary field of study. The Center carries out the most innovative research while also training young researchers from all over the world, especially those from China. In addition to hosting several regular seminars and a colloquium, the Center also frequently hosts larger conferences and workshops on a variety of topics, including topological insulators, big data, and optimization in image processing.

The Center hosted its inaugural events on the afternoon of April 10, 2014, with talks from Prof. Shoucheng Zhang of Stanford University and Prof. Stanley Osher of the University of California at Los Angeles. Before the talks, the establishment of the Center of Mathematical Sciences and Applications was celebrated with a reception attended by Provost Alan Garber, Dean Jeremy Bloxham, Administrative Dean Russ Porter, and a few members of the Evergrande Group, among others. At the lectures, Jeremy Bloxham, Dean of Science, welcomed attendees and congratulated Prof. Shing-Tung Yau, director of the Center of Mathematical Sciences and Applications, for his considerable effort and leadership in estab-

\footnotetext{
* Department of Mathematics, Harvard University E-mail:yau@math.harvard.edu

† The author wishes to thank Sarah LaBauve for her assistance in preparing this article, and for organizing the inauguration ceremony, along with Irene Minde and others in the Department of Mathematics at Harvard University.
}

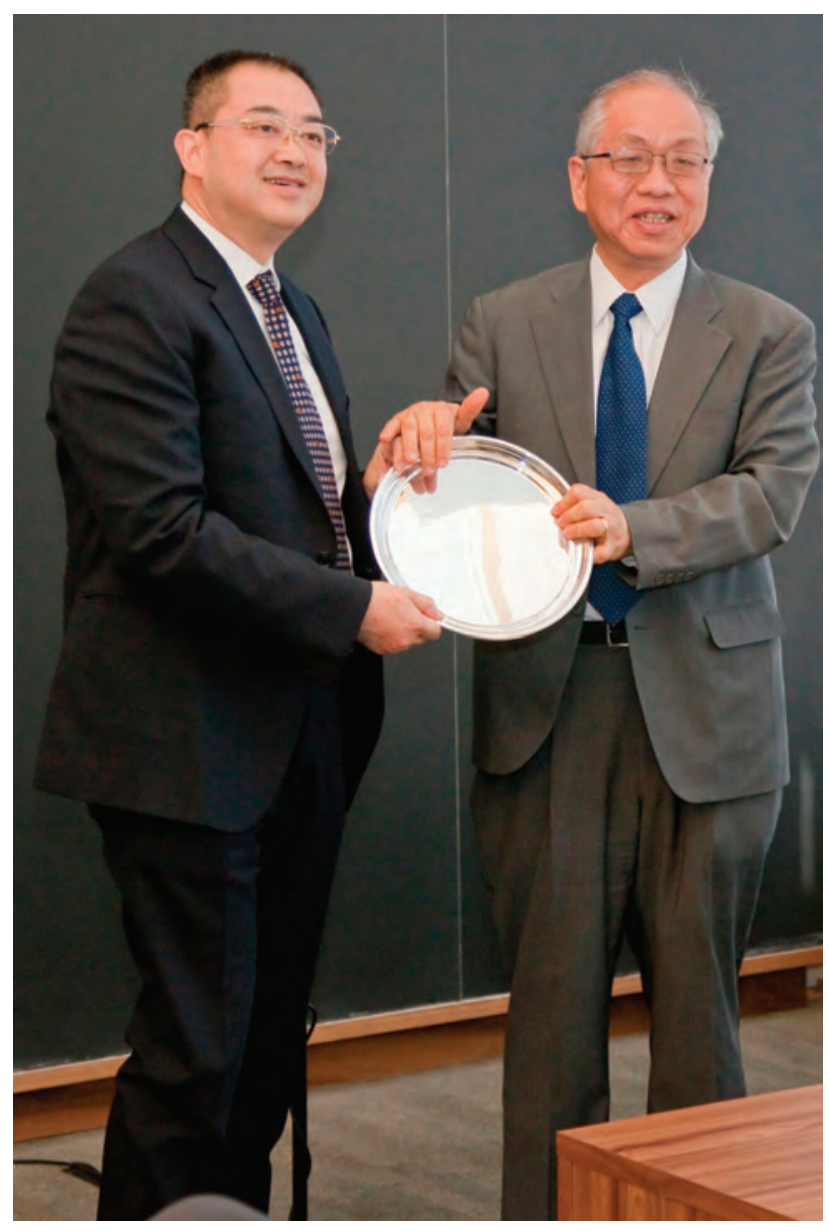

Figure 1. Prof. Shing-Tung Yau, Director of the Center of Mathematical Sciences and Applications, presents Mr. James Xia, CEO of Evergrande, with a gift to celebrate the inaugural events of the Center. 


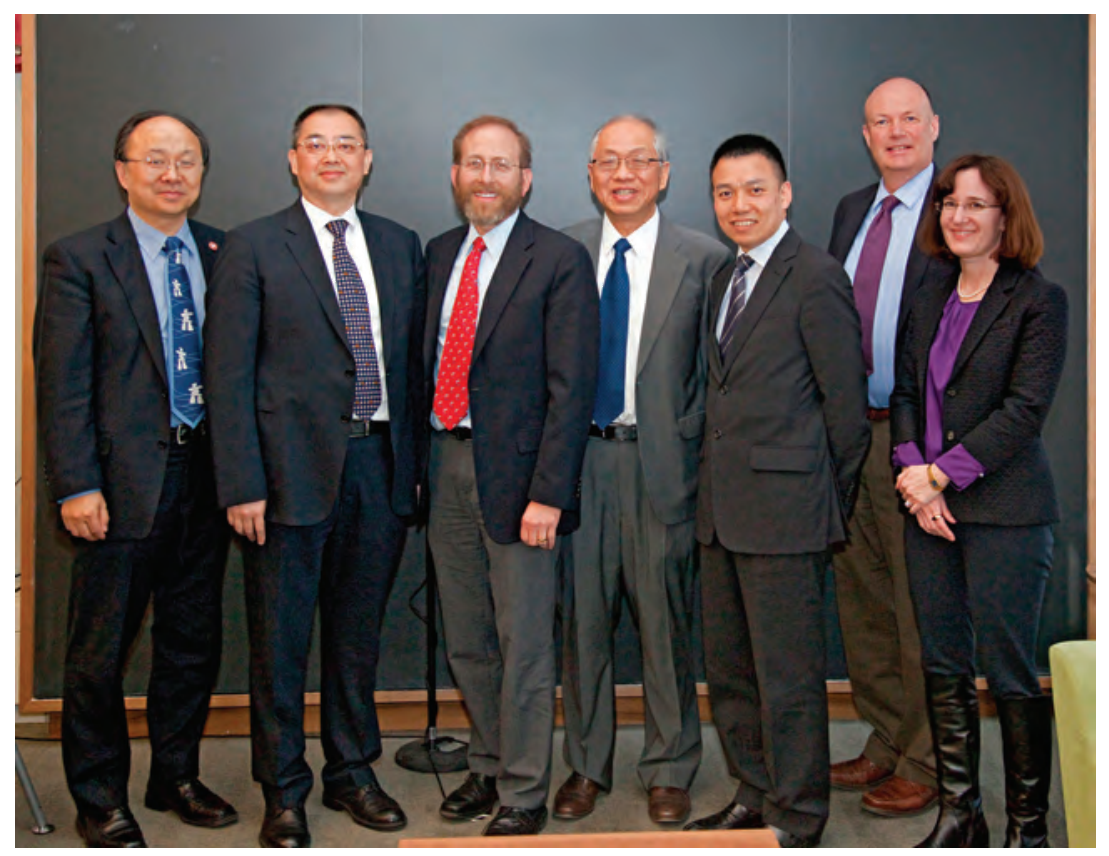

Figure 2. Dean Xiao-Li Meng, Mr. Xia of Evergrande, Provost Alan Garber, Prof. S. T. Yau, Mr. Xu of Evergrande, Dean Jeremy Bloxham, and Dean Nina Zipser together at the inaugural events.

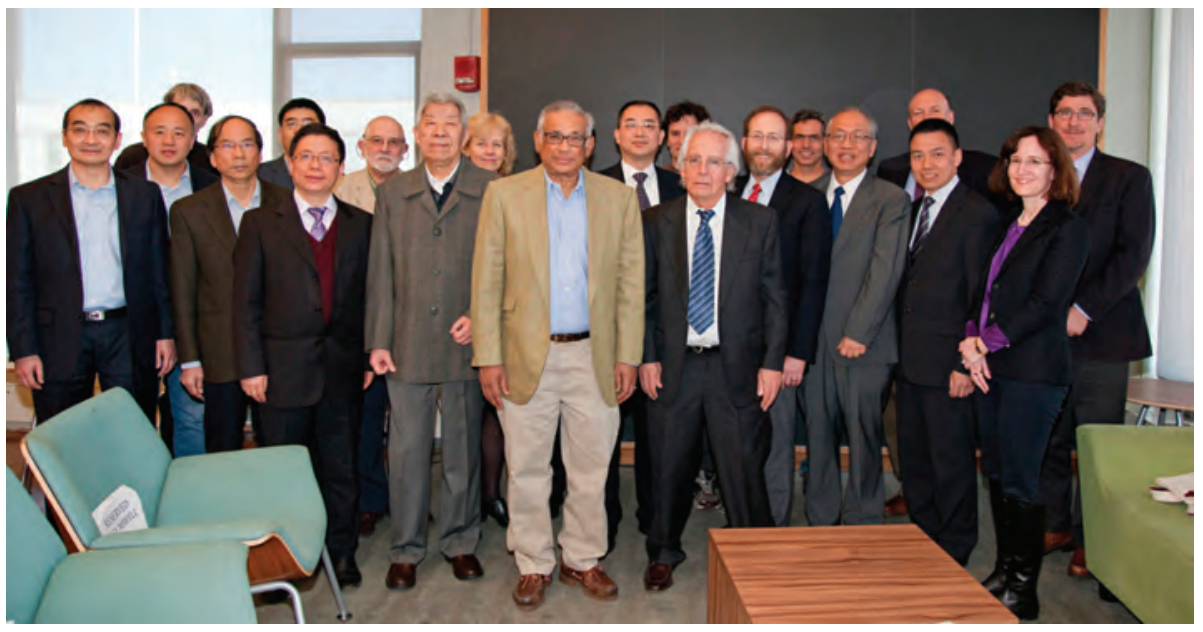

Figure 3. The CMSA's advisory board, scientific board, and member of the Harvard FAS administration together with Mr. Xia and Mr. Xu of Evergrande.

lishing the Center. After, Prof. H. T. Yau of the Harvard Math Department and member of the CMSA Scientific Board introduced Prof. Osher for his talk, "What Sparsity and 11 optimization can do for you."

Prof. Yau then introduced Prof. Zhang and his talk, entitled "Topological insulators, superconductors, and mathematical sciences." Shoucheng Zhang is the J. G. Jackson and C. J. Wood Professor of Physics at Stanford University. His research interests include Theoretical condensed matter physics, including quantum spin Hall effect, topological insulators and superconductors, quantum spintronics, cuprate and pnictide superconductors, superfluidity and magnetism. His prizes and honors include elec- tion to the American Academy of Arts and Sciences in 2011 and receiving the Europhysics Prize in 2010 and the Physics Frontier Prize in 2012.

Stanley Osher is a professor of Mathematics \& the director of Applied Mathematics at the University of California, Los Angeles and also serves as the director of Special Projects at the Institute for Pure and Applied Mathematics. His research involves developing innovative numerical methods to solve partial differential equations, especially those whose solutions have steep gradients, analysis of these algorithms and the underlying P.D.E.'s and applications to various areas of Engineering, Physics and recently, image processing. He is the recipient of many honors 


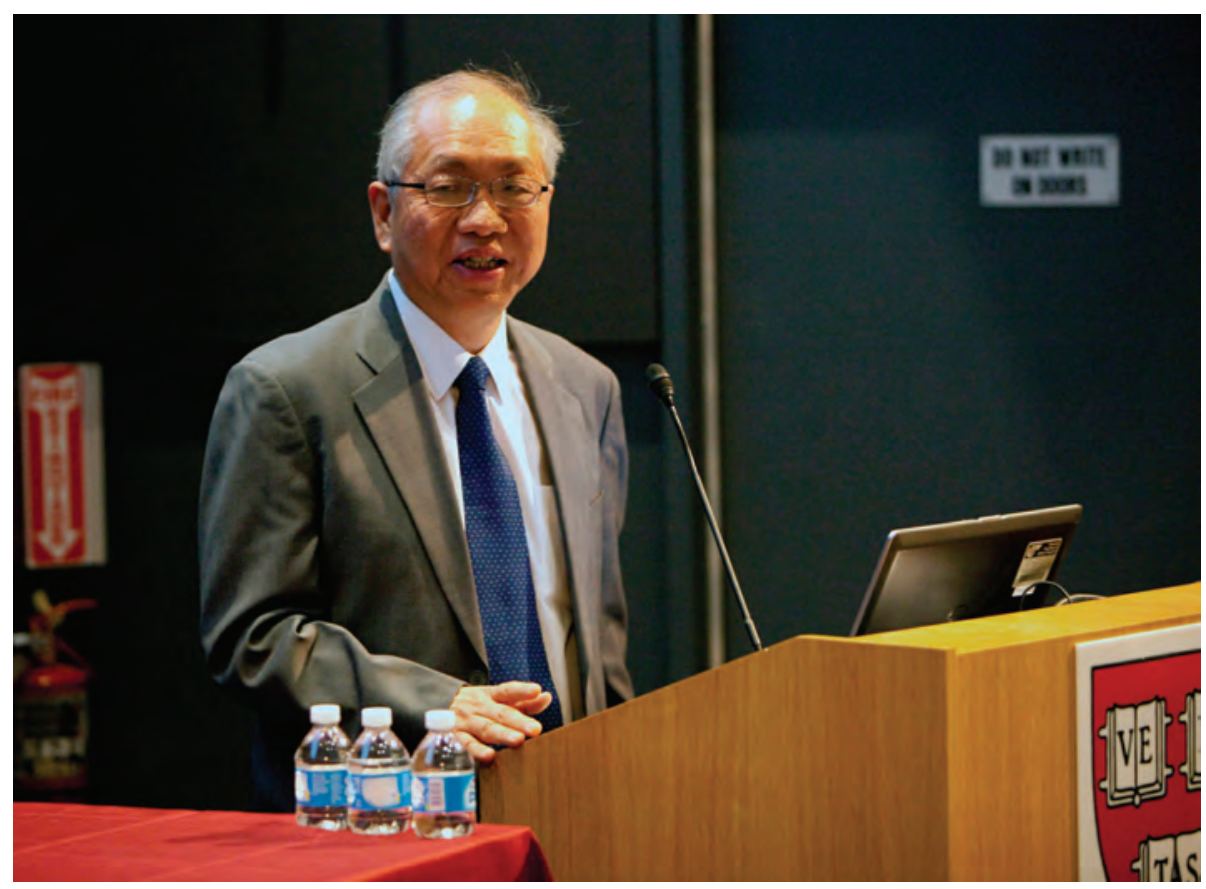

Figure 4. Prof. S. T. Yau introduces the speakers for the center's first public talks.

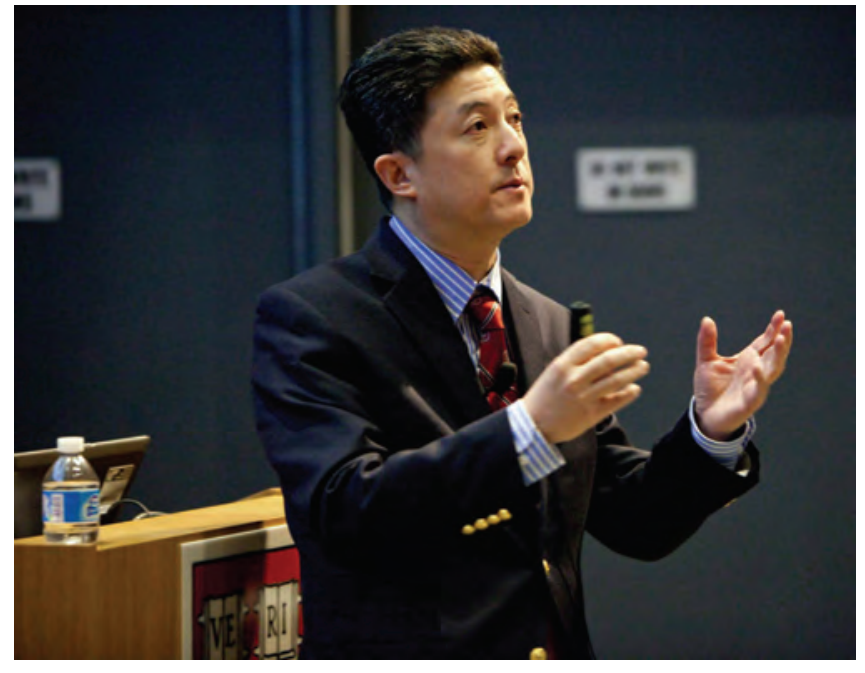

Figure 5. Shoucheng Zhang, the J. G. Jackson and C. J. Wood Professor of Physics at Stanford University, speaks on "Topological insulators, superconductors, and mathematical science."

and awards, including being elected to the American Academy of Arts and Sciences in 2009 and receiving the Carl Freidrich Gauss Prize at the 2014 ICM Meeting.

Following the lectures, members of the Harvard community and mathematical communities at large convened at the nearby Sheraton Commander Hotel for a banquet. Throughout the evening, several members of the Harvard community gave speeches signifying their excitement for the CMSA as well as the other Evergrande centers. Among others, Peter Kron-

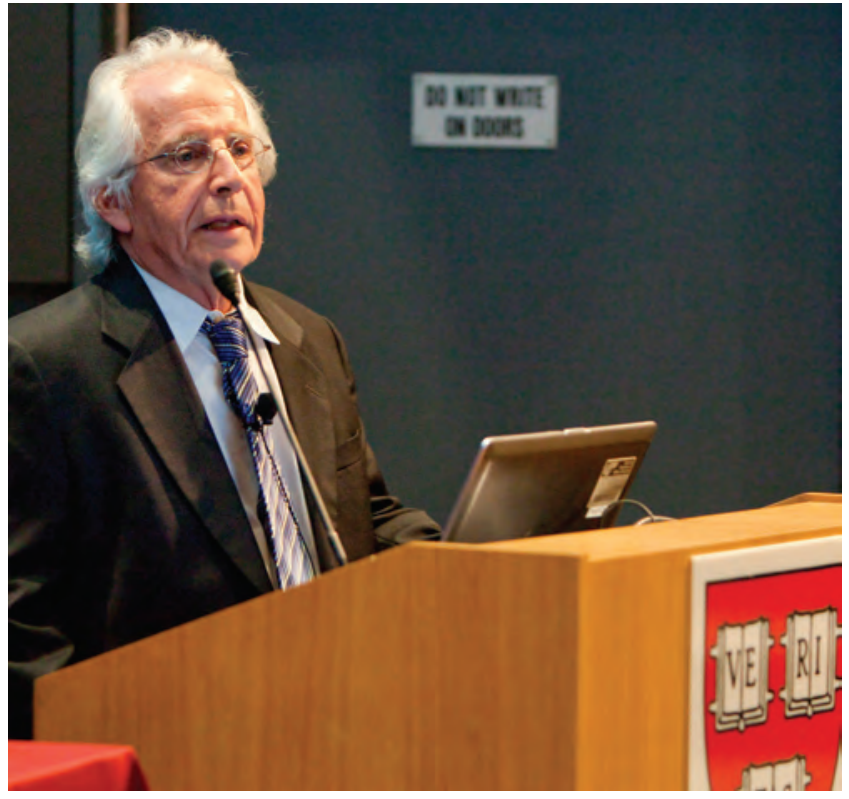

Figure 6. Stanley Osher, Professor of Mathematics at UCLA, speaks on "What sparsity and $\ell_{1}$ optimization can do for you."

heimer (incoming chair of the Harvard Math Department), Vijay Kuchroo (Director of the Evergrande Center for Immunologic Diseases), and Ali Malakwi (Director of the Harvard Center for Green Buildings and Cities) gave short speeches expressing their gratitude to Evergrande for helping Harvard establish this dynamic trio of centers and excitement for what the centers will be able to accomplish both individually and cooperatively. 